\title{
The effect of loading direction on the compressive behaviour of a 3D printed cement-based material
}

\author{
Behzad Zahabizadeh, Vítor M.C.F. Cunha \\ ISISE, Institute of Science and Innovation for Bio-Sustainability (IB-S), Department of Civil \\ Engineering, University of Minho, Guimarães, Portugal.
}

\section{João Pereira}

Innovation Point, Rua de Pitancinhos - Palmeira, 4700-727, Braga, Portugal.

\section{Claúdia Gonçalves}

DST group, Rua de Pitancinhos - Palmeira, 4700-727, Braga, Portugal.

Contacting author: $\underline{\text { b.zahabizadeh@civil.uminho.pt }}$

\begin{abstract}
Nowadays, additive manufacturing is being widely employed in several areas and is starting to be considered for the construction sector amongst the digital construction trend. The advantages that the additive manufacturing techniques can bring over the traditional construction methods are propelling multiple research projects within the field of 3D concrete printing. Technologies used for printing, material compositions and their rheological and mechanical properties are some of the research areas on 3D concrete printing. In this work, it is used a wet extrusion method for printing a cement-based mortar mixture. The compressive behaviour of printed specimens was evaluated based on the direction of loading. The results showed that with a proper printing process and rheological properties, in the case of the current mid-strength matrix, the effect of the layers interfacial behaviour on the compressive behaviour of printed specimens was reduced.
\end{abstract}

Keywords: Digital construction; 3D concrete printing; Wet-extrusion; Layered interface; Compressive behaviour.

\section{Introduction}

Automation within the field of production has started and grown up since the early years of $20^{\text {th }}$ century [1]. However, in the construction industry the process of using automated technologies has grown up slowly when compared with other industries, by e.g. automotive and aeronautics [2]. Nevertheless, even in the construction sector there is a quite strong global competition, thus there is the need of developing new optimized solutions regarding both materials and construction methods. Decreasing the costs (mainly when using complex geometries), increasing the safety and consequently reducing labour related accidents, and also reducing the environmental impacts are some of the main aspects to which additive manufacturing can contribute [2]. 
In recent years, digital construction is one of the approaches that could either mitigate or solve some of the existing problems [1] observed within traditional construction methods $[3,4]$. The 3D concrete printing (3DCP) is a type of rapid prototyping (RP) method based on additive manufacturing (AM) techniques specially intended to be used in conjunction with an integrated design / production philosophy such as in digital construction.

AM processes in the construction industry can show several advantages in comparison with other conventional building methods, which include the development of complex architectural parts without using extra tools or moulds, as well as the ability to add graded functionality to the structures $[2,4-8]$. The potential of decreasing the required man-labour, accelerate the construction process, and decrease the occurrence of possible risks and accidents during the construction are also other aspects benefited from adopting 3D printing technologies in construction [9-11].

AM technique or solid free-form construction is a layer by layer fabrication method for creating objects $[12,13]$. After the design of the digital model through standard CAD packages, the model is exported as a stereolithography file format (STL file), which is an industry standard for faceted models $[2,9,14]$. The 3D printer then uses the STL file to produce the final shape of the 3D model. There are two main categories of 3D printing techniques based on either wet extrusion or powder-based process. Contour crafting (CC) and concrete printing $(\mathrm{CP})$ are two types of 3D printing based on wet extrusion $[15,16]$, while $D$-shape is a powder-based technology [17].

Generally, the material mix design and preparation for 3D printing are focused on optimizing the compatibility and proportion of material with the printer's technological parameters [18]. One of the main issues of using 3D concrete printing, is creating a balance between the rheological and mechanical properties of material and also defining proper parameters for the printing process. The rheological properties include of extrudability (or printability), buildability and open time, which must be controlled to ensure an uniform flow of material during the printing process and a fast setting after extruding [19]. Furthermore, the structural capability and mechanical properties of printed material are another critical issue that must be evaluated in order to check the structural capability and also the quality of the bond between layers [20].

In this work, a 3D printing technology based on wet extrusion of cement-based mixtures is used. The compressive behaviour of the printed material was evaluated after 7 days of curing and compared with conventional mould cast specimens. The influence of the loading direction regarding the printed layers direction on the compressive behaviour was assessed.

\section{Experimental program}

\subsection{Printing technique and equipment}

A small 3D printer developed from the scratch with a printable volume of nearby $0.4 \times 0.4 \times 0.4 \mathrm{~m}$ was used in this study. The printer is comprised of an aluminium frame as a support for a stainless steel funnel with an auger inside. All the movements of printing systems are controlled by stepper motors along $X, Y$, and $Z$ directions, as well as for the rotation of the auger inside the funnel. Figure (1) depicts a general view of the 3D printer.

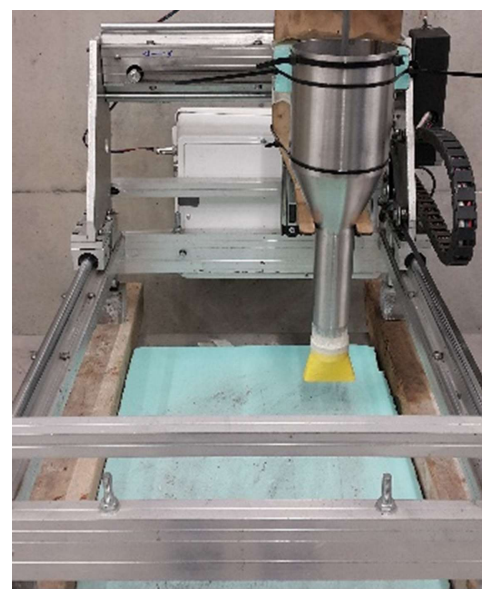

Figure 1. General view of 3D printer

Before starting the extrusion procedure for printing the specimens, the fresh mortar mixture is placed into the funnel. Then, the printing process will start based on predefined parameters for the 3D printer machine and rheological behaviour of the mortar. These parameters include: i) speed of printing, ii) 
flow rate, and iii) geometrical dimensions of the specimens / layers.

During the printing process, the fresh material moves smoothly inside the funnel using the rotation of auger under its own self weight. Finally, the material passes through a customized nozzle to build layer upon layer the desired concrete specimens.

\subsection{Materials and mortar mixture}

The mortar mixture for this study was selected after carrying out several empirical rheological tests on distinct compositions. After evaluating the extrudability, buildability, open time and workability of distinct mixture compositions, the best mortar mixture was selected for assessing its compressive behaviour under distinct loading directions.

A fine sand with a maximum size of $1 \mathrm{~mm}$ was selected based on the smallest dimension of the nozzle $(12 \mathrm{~mm})$. The binder comprised cement CEM I $42.5 \mathrm{R}$, fly ash, and silica fume. For increasing the workability of the mixture, a polycarboxylate based superplasticiser ( $1.3 \%$ by wt. of the total binder) was also added to the mixture. The water to binder ratio $(\mathrm{W} / \mathrm{B})$ for the final mixture was selected equal to 0.31 . Table (1) shows the amounts of the materials used in the final mortar mixture.

Table 1. Mortar mixture compositions

\begin{tabular}{lc}
\hline Material & {$\left[\mathrm{kg} / \mathrm{m}^{3}\right]$} \\
\hline Sand & 1241 \\
\hline Cement & 300 \\
\hline Fly ash & 444 \\
\hline Silica fume & 83 \\
\hline Superplasticiser & 10.8 \\
\hline Water & 260 \\
\hline
\end{tabular}

\subsection{Preparation of printed specimens}

A customized rectangular nozzle with a dimension of $60 \times 12 \mathrm{~mm}$ was used to print the specimens. In this study, the wide of the nozzle was defined to print just one strip in each layer. In this case, the interface is limited to the one between printed layers, thus the effect of the interface between filaments from the same layer was removed.

In order to prepare the specimens for the mechanical characterization, first, three large layered strips were printed, namely with $300 \times 60 \times 50 \mathrm{~mm}, 200 \times 60 \times 50 \mathrm{~mm}$, and $260 \times 60 \times 90$ $\mathrm{mm}$, Figure (2a). The specimens for testing were sawn and then the surfaces were rectified to a dimension of $40 \times 40 \times 80 \mathrm{~mm}$. During the printing process each strip was printed along $X$ direction and the layers were built on top of each other along $Z$ direction, see Figure $(2 b)$.

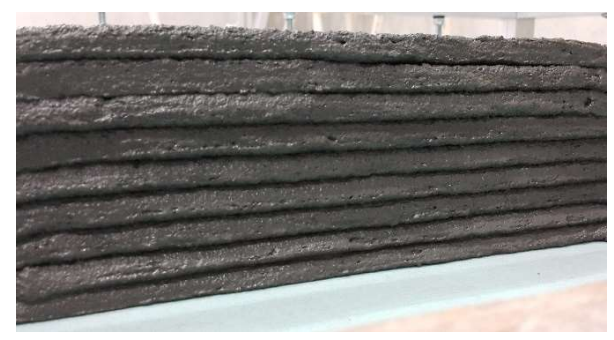

(a)

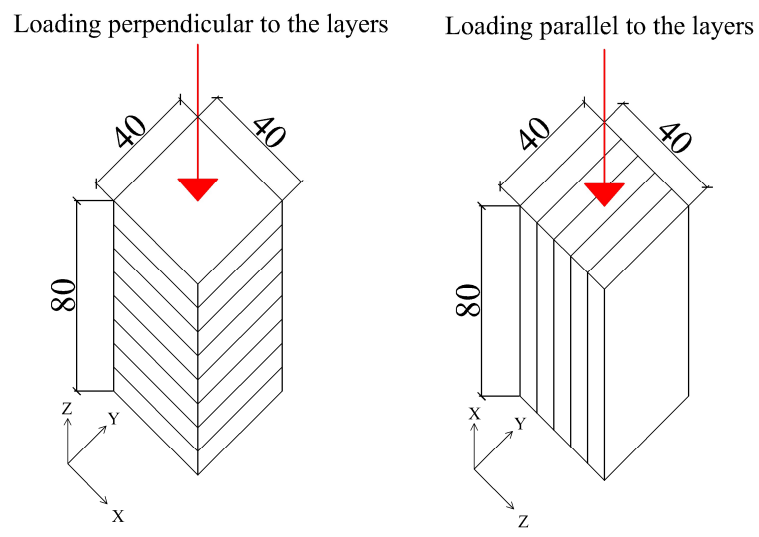

(b)

Figure 2. (a) One sample of printed specimens for compressive strength and elasticity modulus tests and (b) Loading directions relative to printed layer sequence. All dimensions are in $\mathrm{mm}$

Two different series of printed specimens were prepared for mechanical testing. First series were used for assessing the compressive behaviour when loading direction is parallel to the printed layers (series LX) and the second one was used for 
a loading direction perpendicular to the printed layers (series LZ). For each series, five specimens have been used. The specimens for the LX series were extracted from both the $300 \times 60 \times 50 \mathrm{~mm}$ and $200 \times 60 \times 50 \mathrm{~mm}$ strips, on the other hand the specimens for $L Z$ series were extracted from $260 \times 60 \times 90 \mathrm{~mm}$ strip. Figure (2b) shows schematically the loading directions relative to the layers sequences for printed specimens.

Furthermore, five reference prismatic mould-cast specimens (series $\mathrm{MC}$ ) with the same dimensions of the printed ones were also produced. Both the mould-cast and printed specimens were wrapped in plastic sheeting and kept in sealed containers until the desired testing age.

\subsection{Compressive tests}

\subsubsection{Stress - strain relationships}

Compressive strength and stress - strain relationships were assessed in both mould-cast and printed specimens. The test setup for measuring the stress - strain relationships and finally the compressive strength was comprised of a hinged circular steel plate on top of the specimens for applying the load. Three LVDTs were used to measure the deformation. The LVDTs were radially placed with an angle of $120^{\circ}$ between each pair. Figure (3) shows the test setup of the compressive test. The tests were controlled under a displacement rate of $1 \mu \mathrm{m} / \mathrm{s}$.

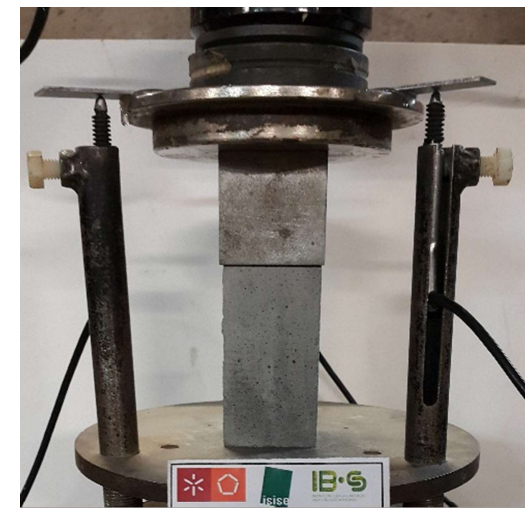

Figure 3. Test setup of compressive test

\subsubsection{Elasticity modulus}

The elasticity modulus tests were previously performed on the same specimens used in the compressive tests for both mould-cast and printed specimens. Before assessing the elasticity modulus and stress - strain curves, one specimen of each series was selected to ascertain the compressive strength. Then based on the results, the linear range of elastic behaviour for the specimens was estimated and the elasticity modulus tests were performed on the remaining specimens using load control and following the recommendations of BS EN 1390-13:2013 [21].

A load cell with a maximum capacity of $300 \mathrm{kN}$ was used. Four LVDTs placed along the four sides of the prismatic specimens were used to measure the axial deformation. The LVDTs were placed on two rectangular rings fastened around the specimens with a distance in between them of $40 \mathrm{~mm}$. Figure (4) shows the test setup for measuring the elasticity modulus.

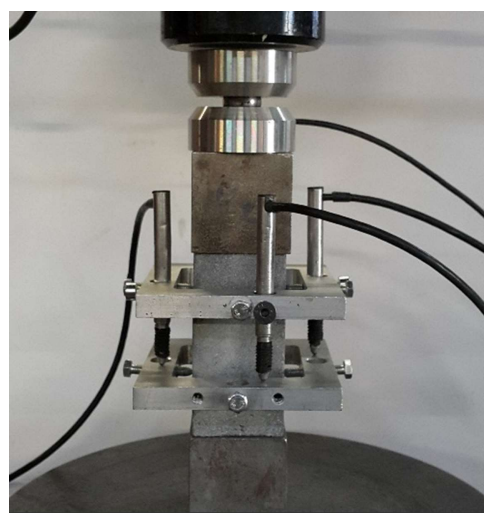

Figure 4. Test setup of elasticity modulus test

\section{Test results and discussion}

\subsection{Stress-strain relationships}

Figure (5) depicts the experimental envelope and average compressive stress - strain relationships obtained for the three studied series, namely LX, LZ and $\mathrm{MC}$, respectively. All specimens were tested at 7 days.

For all series, the compressive stress - strain relationships of the mortar mixtures are characterized by a linear branch almost up to the peak stress, followed by a softening branch. The increase of load causes a fast cracking grow and propagation just before the peak stress is attained. 


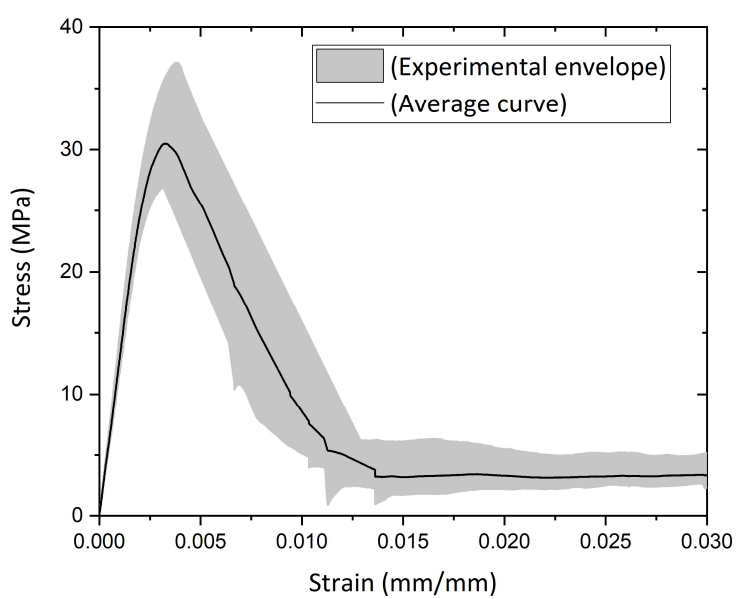

(a)

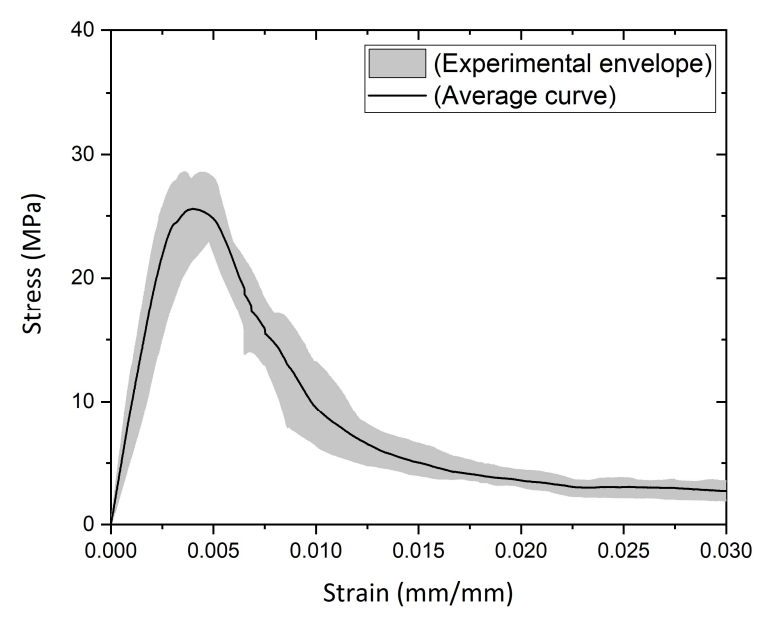

(b)

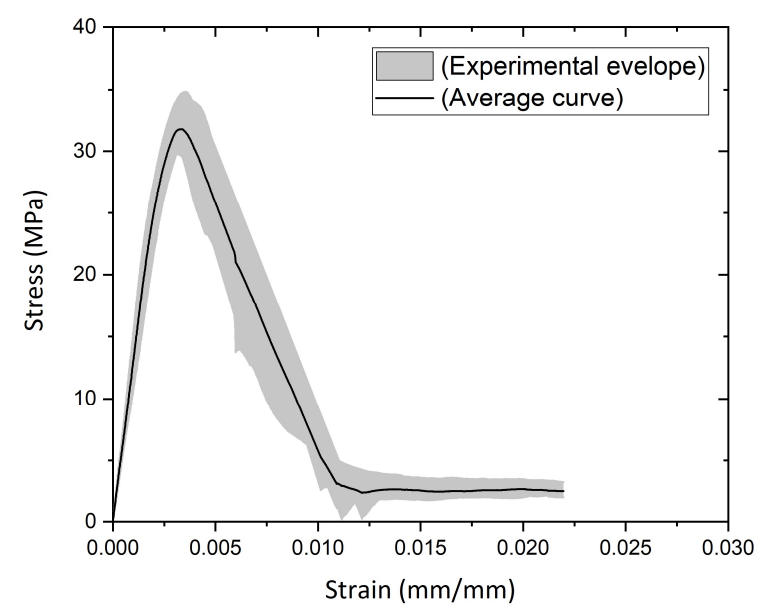

(c)

Figure 5. Stress-strain relationships. (a) $L X,(b) L Z$, and $(c) M C$
Due to the brittle nature of the mixture, in part related to small dimension of the aggregate, at the peak stress, the fracture of the specimens happened suddenly. This brittle behavior was more pronounced on the series with higher compressive strengths, namely LX and MC, which lead to a faster drop of the residual stress and consequently to a steeper softening branch.

The average compressive strengths, $f_{c m}$, were obtained from the five specimens tested in each series and were $30.9,26.7$, and $32.5 \mathrm{MPa}$ for the LX, LZ, and MC series, respectively. Table (2) includes the results of compressive strength for each series with the corresponding coefficient of variation (CoV).

In general, the mould-cast series presented a higher compressive strength and in particular when compared to the $L Z$ series, i.e. when the loading direction is perpendicular to the printed layers. For the latter, a reduction on $f_{c m}$ of approximately $17 \%$ was observed regarding the reference series. Nevertheless, the relative small differences on the $f_{c m}$ demonstrates that by controlling the printing process and rheological properties of the matrix, the anisotropic behavior of the printed specimens may be mitigated.

Table 2. Results of compressive strength $\left(f_{\mathrm{cm}}\right)$ and elasticity modulus (E) with coefficient of variation in $\%$ within parentheses

\begin{tabular}{ccc}
\hline Series & $f_{\mathrm{cm}}$ (MPa) & E (GPa) \\
\hline LX & $30.9(15.16)$ & $27.5(11.73)$ \\
\hline LZ & $26.7(9.23)$ & $27.7(7.78)$ \\
\hline MC & $32.5(5.67)$ & $28.7(10.09)$ \\
\hline
\end{tabular}

\subsection{Elasticity modulus}

Table (2) includes the results of the average elasticity modulus with the respective CoV. The scatter on the average elasticity modulus between series was very low. . An average elasticity modulus of 27.5, 27.7 and 28.7 GPa was observed for the series $L X, L Z$ and MC, respectively. The differences 
between the printed and mould cast series was lower than $3.5 \%$.

\subsection{Fracture modes}

The fracture of the specimens under compressive loads with the localization of the macro-cracks has occurred suddenly at the peak stress.

Furthermore, the dominant fracture mode in the LX and MC series included a wide diagonal crack with some narrower cracks parallel to the specimen's longitudinal axis. On the other hand, in the LZ series, the dominant fracture mode was composed of an hourglass crack pattern from the opposite edges of the specimens. However, a single wide diagonal crack was also observed in some specimens of the LZ series. Figure (6) shows the typical fracture modes observed for each series. The arrows on the printed specimens show the direction of printed layers, i.e. of the layer's interfaces.

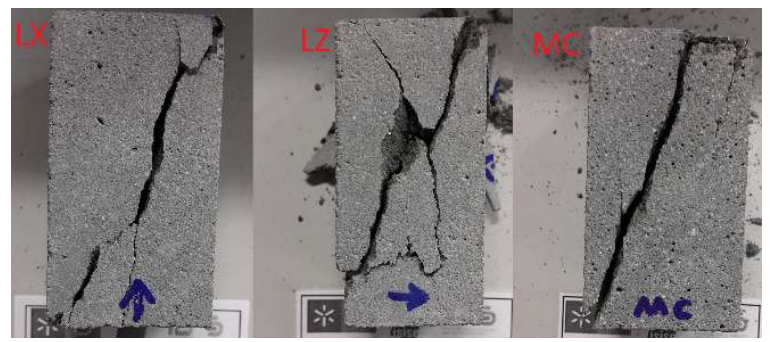

Figure 6. Fracture modes

In general, the interface of the layers did not had a significantly influence on the fracture mechanisms, even though some secondary cracking is observed along the direction of the layers' interface surfaces. Therefore, the material's rheological properties in terms of extrudability lead to a reasonable bond strength in between layers, which was achieved for this mid-strength mortar mixture. In the majority of the specimens, the fracture occurred exclusively across the matrix layers instead of along the layer's interface.

\subsection{Influence of the loading direction on the compressive behaviour}

Figure (6) resumes the results of the compressive strength and elasticity modulus of both printed and mould-cast specimens using a column chart.
Comparing the results of the three different series of specimens shows that the highest compressive strength and also elasticity modulus are related to the mould-cast (MC) series. Regarding the printed specimens, the compressive strength of series $L X$ under parallel loading towards the layers' direction is higher than the series LZ loaded perpendicular to the layers' direction. No significant variations were observed on the elasticity modulus, which indicates that the layers' interfacial bond strength is higher than the stress levels that the specimens are subjected during this test.

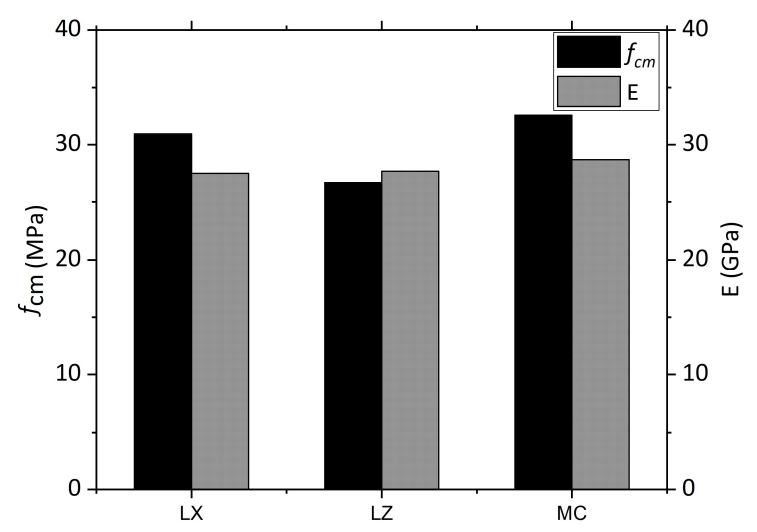

Figure 7. Average values of the compressive strength $\left(f_{c m}\right)$ and elasticity modulus $(E)$.

\section{Conclusions}

In this work, the influence of the loading direction regarding the printing direction on the compressive behaviour of mid-strength printed specimens was investigated. A mortar mixture with $300 \mathrm{~kg} / \mathrm{m}^{3}$ of cement was used. Two series of printed specimens and loaded in two different directions regarding the printing direction were studied. An additional reference series with cast specimens was used. The compressive behaviour was assessed at 7 days.

In general, a good printing homogeneity was obtained; in particular, regarding extrusion and interface bond between the printed layers. The differences on the compressive strengths observed for the printed and mould-cast specimens were relatively reduced. Nonetheless, it was observed a strength reduction for the printed series, in particular for the one where the layers' direction was perpendicular to the load. Furthermore, in general, the mid-strength printed specimens 
fractured across the matrix itself instead of along the interface surfaces of the printed layers.

Finally, deeper knowledge about the effect of loading directions on the compressive behaviour of printed specimens is required. Moreover, it is necessary to ascertain the influence of the compressive strength on the commanding fracture mechanisms.

\section{References}

[1] Khoshnevis B. Automated construction by contour crafting-related robotics and information technologies. Automation in Construction 2004; 13: 5-19.

[2] Buswell RA, Soar RC, Gibb AGF, et al. Freeform Construction: Mega-scale Rapid Manufacturing for construction. Automation in Construction 2007; 16: 224231.

[3] Lim S, Buswell RA, Le $\pi$, et al. Developments in construction-scale additive manufacturing processes. Automation in Construction 2012; 21: 262 268.

[4] Perrot A, Rangeard D, Pierre A. Structural built-up of cement-based materials used for 3D-printing extrusion techniques. Materials and Structures 2016; 49: 1213-1220.

[5] Buswell RA, Soar RC, Pendlebury M, et al. Investigation of the potential for applying freeform processes to construction. In: IN: Proceedings of the 3rd International Conference on Innovation in Architecture, Engineering and Construction (AEC). Rotterdam, Netherlands, pp. 141-150.

[6] Hopkinson N, Gao Y, McAfee DJ. Design for environment analyses applied to rapid manufacturing. Proceedings of the Institution of Mechanical Engineers, Part D: Journal of Automobile Engineering 2006; 220: 1363-1372.

[7] Hopkinson N, Dicknes P. Analysis of rapid manufacturing-using layer manufacturing processes for production. Proceedings of the Institution of Mechanical Engineers, Part C: Journal of Mechanical Engineering Science 2003; 217: 31-39.

[8] Lim S, Buswell R, Le T, et al. Development of a viable concrete printing process. Seoul, South Korea, pp. 665-670.

[9] Feng $P$, Meng $X$, Zhang $H$. Mechanical behavior of FRP sheets reinforced 3D elements printed with cementitious materials. Composite Structures 2015b; 134 : 331-342.

[10] Pegna J. Exploratory investigation of solid freeform construction. Automation in Construction 1997; 5: 427-437.

[11] Perkins I, Skitmore M. Three-dimensional printing in the construction industry: A review. International Journal of Construction Management 2015; 15: 1-9.

[12] Stavric M, Sidanin P, Tepavcevic B. Architectural Scale Models in the Digital Age: Design, Representation and Manufacturing. AMBRA, 2013.

[13] Ford S, Despeisse M. Additive manufacturing and sustainability: an exploratory study of the advantages and challenges. Journal of Cleaner Production 2016; 137: 1573-1587.

[14] Pham D., Gault R. A comparison of rapid prototyping technologies. International Journal of Machine Tools and Manufacture 1998; 38: 1257-1287.

[15] Khoshnevis B, Bukkapatnam S, Kwon H, et al. Experimental investigation of contour crafting using ceramics materials. Rapid Prototyping Journal 2001; 7: 32-42.

[16] Lim S, Le T, Webster J, et al. Fabricating construction components using layer manufacturing technology. Loughborough University, http://oro.open.ac.uk/37889/ (2009, accessed 29 May 2018). 
[17] Cesaretti G, Dini E, De Kestelier X, et al. Building components for an outpost on the Lunar soil by means of a novel 3D printing technology. Acta Astronautica 2014; 93: 430-450.

[18] Ma G, Wang L. A critical review of preparation design and workability measurement of concrete material for largescale 3D printing. Frontiers of Structural and Civil Engineering 2017; 12: 382-400.

[19] Weng Y, Lu B, Tan MJ, et al. Rheology and Printability of Engineered Cementitious Composites-A Literature Review, https://dr.ntu.edu.sg/handle/10220/41821 (2016, accessed 7 May 2018).

[20] Le TT, Austin SA, Lim S, et al. Hardened properties of high-performance printing concrete. Cement and Concrete Research 2012b; 42: 558-566.

[21] BS EN 12390-13. Testing hardened concrete - determination of secant modulus of elasticity in compression. 